\title{
Obstructive anuria of adults in the region of eastern morocco: epidemiological, diagnosis and therapeutic aspects. retrospective two center study of 44 cases
}

\begin{abstract}
Purpose: To analyze the aspects of obstructive anuria in adults

Materials and Methods: A two center retrospective study over a period of 4years (20112014) compiled 44 patients in two urology departments in the region of eastern Morocco. Many data were collected. We studied two groups: the first group of stone formers patients $(n=16)$ and the second group of neoplastic patients $(n=28)$. A comparison between the two groups was performed.

Results: The mean age was 61years with a sex ratio of 1.3 . The main symptoms were anuria (50\%) and back pain (50\%). The obstruction was neoplastic in 28 cases, and 16 cases of stones. A renal urine diversion performed in 11 patients $(25 \%)$, either by percutaneous nephrostomy in 26 cases $(59.1 \%$ ) or by a double-J ureteral probe in 13 cases $(29.5 \%)$. There was 3 deaths $(6.8 \%)$ and 9 cases of chronic renal failure $(20.45 \%)$.

Discussion: To our knowledge, this is the first epidemiological and analytical study on obstructive anuria carried out in the region of eastern Morocco. Urgent diagnosis and therapeutic management are requested to prevent metabolic consequences. Helical CT scan without enhancing contrast is a promising technique for anuria's exploration. It is indicated whenever ultrasonography and standard radiography cannot determine the cause of anuria. Antegrade opacification by the nephrostomy probe and retrograde ureteropyelography (UPR) can be helpful to visualize the nature of the obstacle and its headquarters, but these two examinations are less practiced since the advent of the CT scan.
\end{abstract}

Conclusion: Obstructive anuria is an urological emergency that involves immediate and functional prognosis of the kidney. Its management is closely related to the etiology of the obstruction which also determines the prognosis.

Keywords: anuria, obstruction, stones, cancer
Volume 5 Issue I - 2017

Fahd khalil

Urology department, Mohammed the sixth university hospital, Morocco

Correspondence: Fahd Khalil, Associate professor of urology Urology department, Mohammed the sixth university hospital, Mohammed the first university, OUJDA, Morocco, Email drf.khalil@hotmail.fr

Received: March 31, 2017 | Published: June 26, 2017

\section{Introduction}

Obstructive anuria is defined by the absence or defective excretion of less than $200 \mathrm{ml}$ of urine in 24 hours, due to an acute obstruction of the upper urinary tract. ${ }^{1}$ Obstruction can occurs bilaterally or in anatomical or functional single kidney. ${ }^{2}$ This situation requires early diagnosis and urgent care to prevent metabolic disorders. ${ }^{3}$ The purpose of this study is to investigate the epidemiological, clinical, diagnostic, treatment and outcome of obstructive anuria according to its stone or neoplastic nature in order to improve its management and avoid chronic renal failure.

\section{Materials and methods}

This is a retrospective study of 44 patients diagnosed and treated for obstructive anuria in two urology departments of the region of eastern Morocco, for a period ranging from 2011 to 2014. The study included patients older than 18years. Excluded criterias were incomplete records and anurias having as origin other than stones or pelvic cancers (retroperitoneal fibrosis, ureteral stenosis by several infections (tuberculosis, schistosomiasis, mycotic) or malformation disease. Ultrasonography and standard radiography of the urinary tract was performed at the patient's admission in the emergency department, followed by a CT scan or magnetic resonance imaging. A complete biological panel including creatinine, serum potassium and bicarbonates ions were consistently requested. The Variables analyzed were: sex, age, background, the consultation period, symptoms, diagnostic tests, aetiology, treatment modalities and outcome. Patients were divided into two groups. The first group of stone patients $(n=16)$, and the second group of cancer patients $(n=28)$. Statistical analysis was made using SPSS 20.0 with a significance level of $p<0.05$. Finally, our results were compared with literature data through a systematic review of pubmed database.

\section{Results}

Patients were mainly 25 men and 19 women. The sex ratio was 1.3 with male predominance. The average age was 61years (range 18 to 86 years). The average time of consultation was 7.6 days (range 1 day to 25 days). Personal history of patients was dominated by hypertension (36.4\%), diabetes (25\%), pelvic radiotherapy $(13.6 \%)$, chemotherapy $(18.2 \%)$, stone urinary history $(20.5 \%)$ and cancer $(43.2 \%)$. The reason for consultation was typically anuria in 22 cases $(50 \%)$, oligoanuria in 22 cases $(50 \%)$, lumbar pain in 22 cases $(50 \%)$, abdominal pain in 10 cases $(22.7 \%)$, symptoms of lower urinary tract in 17 cases $(38.6 \%)$, fever in 8 cases $(18.2 \%)$, dyspnea in 18 cases $(40.9 \%)$, consciousness disorders in 15 cases $(34.1 \%)$, impaired general condition in 16 cases $(36.3 \%)$ and hematuria in 12 cases $(27.3 \%)$ (Table 1$)$. The renal pelvis dilatation was found on ultrasound in all cases with cortico-medullary dedifferentiation in 10 cases $(25.6 \%)$. The obstruction had occurred 
in a single kidney in 7 patients $(16 \%)$. A nephrectomy of the opposite kidney was made in 4 cases $(9.1 \%)$, a non-functional kidney diagnosed in 3 cases $(6.8 \%)$ and was agenesic in one case $(2.3 \%)$. In 37 patients $(84 \%)$, obstruction was bilateral. The diagnosis was performed by ultrasonography in 22 cases (50\%), a non-contrast CT scan in 21 cases (45.4\%), abdominopelvic MRI in one case $(2.3 \%)$, and retrograde ureteropyelography in another case. Renal insufficiency was severe in 37 cases $(84 \%)$ and moderate in 7 cases $(16 \%)$. Metabolic acidosis was found in 11 cases $(25 \%)$ associated with hyperkalemia in 20 cases $(45 \%)$ and severe hyperkalemia in 8 cases $(18 \%)$ (Table 2$)$. Urine culture was positive in 8 cases $(18.2 \%)$.

Table I Main symptoms and associated signs of anuria

\begin{tabular}{lll}
\hline & N & $\%$ \\
\hline Anuria & 22 & 50 \\
Oligo-Anuria & 22 & 50 \\
Flank Pain & 22 & 50 \\
Abdominal Pain & 10 & 22,7 \\
Fiever & 8 & 18,2 \\
Hématuria & 12 & 27,3 \\
Dyspnea & 18 & 40,9 \\
Poor general condition & 16 & 36,3 \\
Altered Consciousness & 15 & 34,1 \\
Low urinay tract symptoms & 17 & 38,6 \\
Urinary Infection & 8 & 17 \\
\hline
\end{tabular}

By screening the aetiologies, the first two causes identified were pelvic cancer in 28 cases $(63.6 \%)$ and nephrolithiasis and/or ureteral stones in 16 cases $(36.4 \%)$ (Figure 1). Metabolic disorders were major requiring the use of renal diversion in 11 cases $(25 \%)$, either by percutaneous nephrostomy tube in 26 cases $(59.1 \%)$ or by a double $\mathrm{J}$ ureteral stent in 13 cases (29.5\%). The etiological treatment was instituted whenever possible taking into account the cancer level and following the scientific recommendations (French association of urology, European association of urology). Therapeutic results are summarized in Table 3. The two patients groups were compared and the statistical significance analyzed (Table 4). After a mean followup of 19.5 months (3 months- 36months), the overall outcome was good with resumption of normal renal function in 35 patients $(79.5 \%)$ Chronic renal insufficiency was developed in 9 cases $(20.4 \%)$. We had three deaths in our series $(6.8 \%)$.

Table 2 Metabolic disorders related to obstructive anuria

\begin{tabular}{lll}
\hline & N & $\%$ \\
\hline $\begin{array}{l}\text { Creatinine Clearence } \\
<15\end{array}$ & 37 & 84 \\
{$[15-30]$} & 7 & 16 \\
{$[30-45]$} & 0 & 0 \\
Kaliemia & & \\
Hyperkaliemia & 20 & 45 \\
Severe Hyperkaliemia & 8 & 18 \\
Alcaline Réserve $<25 \mathrm{mmol} / \mathrm{I}$ & $\mathrm{II}$ & 25
\end{tabular}

Table 3 Treatment panel

\begin{tabular}{lll}
\hline Treatment & N & $\%$ \\
\hline Hemodialysis & II & 25 \\
Antibiotics & I I & 25 \\
Percutaneous nephrostomy & 26 & 59, I \\
Double J probe & I3 & 29,5 \\
No surgery & I & 2,3 \\
\hline
\end{tabular}

Table Continued..

\begin{tabular}{lll}
\hline Treatment & N & $\%$ \\
\hline $\begin{array}{l}\text { Refused for surgery } \\
\text { Stone }\end{array}$ & $\mathrm{I}$ & 2,3 \\
Alcalinisation & 5 & 31,25 \\
J + extra-corporal shock waves & 3 & 18,75 \\
Ureteroscopy & 2 & 12,5 \\
Open surgery & 4 & 25 \\
No surgery & $\mathrm{I}$ & 6,25 \\
Refused for surgery & $\mathrm{I}$ & 6,25 \\
Pelvic Cancer & & \\
Radio-chemotherapy & 5 & 17,85 \\
Hormonotherapy & 8 & 28,57 \\
Surgery & 10 & 35,71 \\
Curative & 3 & 10,71 \\
Palliative & 7 & 25 \\
\hline
\end{tabular}

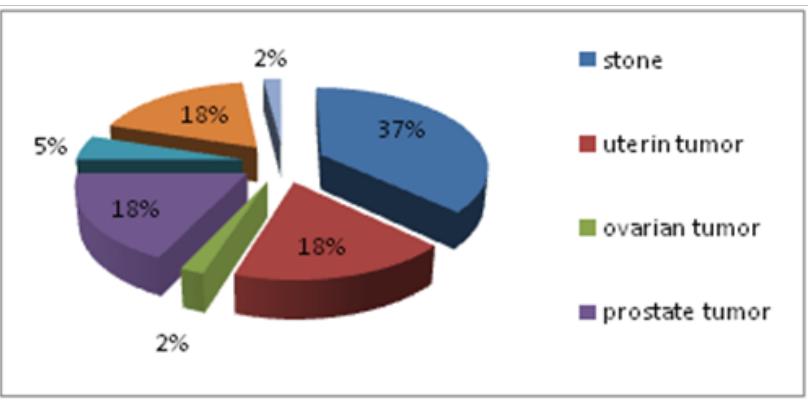

Figure I Aetiologies of obstructive anuria

\section{Discussion}

Obstructive anuria represents 2 to $10 \%$ of cases of acute renal failure. ${ }^{3}$ In Morocco, it is the origin of $8.7 \%$ of acute renal failure. ${ }^{4}$ To our knowledge, this is the first epidemiological and analytical study on obstructive anuria carried out in the region of eastern Morocco. It is a medical and surgical emergency that can be life-threatening. ${ }^{1,4,5}$ Urgent diagnosis and therapeutic management are requested to prevent metabolic consequences. ${ }^{6}$ The diagnosis of obstructive anuria is mainly clinical based on the absence of urine and bladder globe. ${ }^{1,6}$ Imaging tools are used to specify the etiology and to locate exactly the obstruction. ${ }^{3,7}$ Ultrasonography is the gold standard imaging tool. ${ }^{2,6}$ It was performed at first intention in our series. It seeks uretero-renal pelvis dilatation reflecting the level of obstruction, gives an idea on renal parenchyma thickness and contralateral kidney condition and can guide a possible implementation of a percutaneous nephrostomy tube. $^{2,7}$ The standard radiography can detects stones, but its poor sensitivity can detect only $45 \%$ to $59 \%$ of ureteral calculi. ${ }^{6,7}$ Helical CT scan without enhancing contrast is a promising technique for anuria's exploration. ${ }^{2,7}$ It is indicated whenever ultrasonography and standard radiography cannot determine the cause of anuria. CT scan shows stones of $2 \mathrm{~mm}$ and the main obstructive etiologies. ${ }^{8}$

This examination was carried out only in 21 cases for the diagnosis $(45.4 \%)$ but was not always available in emergency. ${ }^{6-8}$ The MRI allows the exploration of the upper urinary tract in three planes without contrast enhancing or patient irradiation, but is poorly available in the urgent setting, and was performed in one case of this study $(2.3 \%)$. Antegrade opacification by the nephrostomy probe and retrograde ureteropyelography (UPR) can be helpful to visualize the nature of the obstacle and its headquarters, but these two examinations are less practiced since the advent of the CT scan. ${ }^{3,5}$ In our study, the UPR was performed in one case. In this study, obstruction appeared 
bilaterally in the majority of our patients, unlike the Tunisian data set for which the mechanism involved was a single kidney obstruction in most cases. ${ }^{5}$ The etiologies were dominated by pelvic cancers (bladder cancer, prostate cancer, digestive and gynecological) and urolithiasis in our series as in the literature ${ }^{1,3,9}$. In addition, other causes are listed in the literature including retroperitoneal fibrosis, acute hydronephrosis by ureteropelvic junction obstruction, iatrogenic ureteral injuries and other rare causes of ureteral stenosis such as tuberculosis or schistosomiasis. ${ }^{7,8}$ It is essential to note that the impact of the obstruction should be evaluated previously, especially metabolic and electrolyte disturbances. ${ }^{8}$ When these problems are major, urgent kidney diversion is strongly recommended. ${ }^{5,8}$ This was performed in $25 \%$ of cases in our series.

Table 4 Comparison of the two groups

$\begin{array}{ll} & \text { Neoplastic Anuria } \\ \text { Main Age } & 64 \text { ans } \\ \text { Time Prior consultation } & 10,2 \text { jours } \\ \text { Sex Ratio } & 1,54 \\ \text { True Anuria } & 10(35,7 \%) \\ \text { Poor general condition } & 50 \% \\ \text { Dialysis } & 9(32,14 \%) \\ \text { Nephrostomy } & 22(78,5 \%) \\ \text { Double J probe } & 3(10,7 \%) \\ \text { Severe infection } & 3(10,71 \%) \\ \text { Chronic renal failure } & 7(25 \%) \\ \text { Death } & 2 \\ \text { Mortality } & 7,14 \%\end{array}$

\begin{tabular}{ll} 
Stone Anuria & Statistical Significance $(\mathbf{P})$ \\
57 ans & $\mathrm{P}=0,69$ \\
2,62 jours & $\mathrm{P}=0,1$ \\
$\mathrm{I}$ & $\mathrm{P}=0,35$ \\
$12(75 \%)$ & $\mathrm{P}=0,013$ \\
$12 \%$ & $\mathrm{P}=0,034$ \\
$2(12,5 \%)$ & $\mathrm{P}=0,138$ \\
$4(25 \%)$ & $\mathrm{P}=0,001$ \\
$10(62,5 \%)$ & $\mathrm{P}=0,001$ \\
0 & $\mathrm{P}=0,247$ \\
$2(12,5 \%)$ & $\mathrm{P}=0,25 \mathrm{I}$ \\
\hline & $\mathrm{P}=0,7$ \\
$6,25 \%$ & $\mathrm{P}=0,65$
\end{tabular}

The goal of therapeutic management is to remove the obstruction, to manage complications and metabolic disorders, and finally to treat the etiology. ${ }^{8,9}$ The type of urinary diversion to propose differs from the etiology. ${ }^{10}$ For the original stone obstruction, retrograde drainage by the double J probe represents the first line of treatment, achieved in $62.5 \%$ of cases in our series, except in cases of purulent retention and if the size of ureteral calculus is $>2 \mathrm{~cm}$, where we used a percutaneous nephrostomy tube. ${ }^{5,10,11}$ During the establishment of a ureteral catheter, ureteroscopy with fragmentation of obstructive calculation could be considered. ${ }^{12,13}$ But in our series, ureteroscopy was performed in a second time. Certainly this percutaneous nephrostomy has many advantages including the ability to conduct opacification, lithotripsy and treatment of ureteropelvic junction obstruction by endopyelotomy. ${ }^{9,10,14}$

The immediate lithotomy is related to a high morbidity rate. In addition, surgical nephrostomy has a high overall mortality $(6 \%)$ compared with percutaneous nephrostomy $(0.2 \%) .{ }^{15}$ For pelvic cancers, nephrostomy is the most appropriate treatment, performed in $78.5 \%$ of cases in our series. It has a low failure rate, reduces morbidity, and can be performed under local anesthesia. ${ }^{16}$ On the other hand, the tortuous ureter traject induces by neoplastic process and locoregional invasion reduces the success rates of the ureteral catheter ${ }^{16,17}$ The use of bilateral ureterostomy is exceptional in the literature. ${ }^{18}$ The prognosis also differs according to etiology. Mortality varies from 6.2 to $25 \%$ in the literature ( $6.8 \%$ in our series). ${ }^{1,3,16}$ Stones anuria is globally related with good prognosis (mortality of $6.25 \%$ ), with the possibility of an effective etiological treatment and a high rate of return of a normal renal function. Neoplastic anuria is often with poor prognosis ( $7.14 \%$ of mortality). The factors involved are chronic renal failure and the advanced nature of the underlying disease. ${ }^{5,18}$

\section{Conclusion}

The diagnosis of obstructive anuria is focused on clinical statement, confirmed by ultrasonography. The etiologies are dominated by pelvic cancers and stones in our context. We strongly recommend the use of ureteral double $\mathrm{J}$ probe for initial urinary drainage for lithiasic obstructions and nephrostomy tube for neoplastic pathologies. The

anuria of stone origin has a good prognosis compared to the neoplastic origin.

\section{Acknowledgments}

None.

\section{Conflicts of interest}

Author declares there are no conflicts of interest.

\section{Funding}

None.

\section{References}

1. Bennani S, Debbagh A, Joual A. Anuries obstructives. A propos de 30 cas. Ann Urol. 1995;29:159-162.

2. Hubert J, Descotes JL, Bellin MF. Imagerie et lithiase urinaire. Prog Urol. 2003;13:993-1021.

3. Rakototiana AF, Ramorasatal AJC, Rakotomena SD, et al.Anurie obstructive: à propos de 42 cas consécutifs. Revue d'AnesthésieRéanimation et de Médecine d'Urgence. 2011;3(1):32-34.

4. El Khayat SS, Bourial M, Benghanem M, et al. L'insuffisance rénale aiguë vue par les néphrologues: enquête nationale. Néphrologie \& Thérapeutique. 2014;10(5):356.

5. Nouira Y. L'anurie lithiasique: étude clinique de 48 patients et comparaison entre le drainage antérograde et rétrograde des cavités rénales en urgence. J Maroc Urol. 2006;1(2):13-15.

6. Shokeir AA, Shoma AM, Mosbah A, et al. Noncontrast Computed Tomography In Obstructive Anuria: A Prospective Study. Urology. 2002;59(6):861-864.

7. Joual A, Dakir M, El Mrini M. L'anurie calculeuse A propos de 25 cas . Annales d'urologie. 1997;31(4):191-194.

8. Amanullah, Khan G, Lal S, et al.Calculus anuria and its remedy. $J$ Ayub Med Coll Abbottabad . 2010;22(1):112-114. 
9. El-Sheemy MS, Shouman AM, Shoukry AI, et al. Selection criteria for initial urinary drainage in relation to definitive stone management in obstructive calcular anuria with acute renal failure in children less than 4 years in age Eur Urol Suppl. 2014;13;e556.

10. Ahmad I, Saeed Pansota M, Tariq M, et al. Comparison Between Double J (DJ) Ureteral Stenting and Percutaneous Nephrostomy (PCN) in Obstructive Uropathy. Pak J Med Sci. 2013;29(3):725-729.

11. ElSheemy MS, Shouman AM, Shoukry AI, et al. Ureteric stents vs percutaneous nephrostomy for initial urinary drainage in children with obstructive anuria and acute renal failure due to ureteric calculi: a prospective, randomised study. BJU . 2015;115(3):473-479.

12. Abdel-Kader MS. Management of calcular anuria in adults caused by ureteric stones: By using of ureteroscopy and holmium laser. Arab J Urol. 2011;9(3):179-183.

13. Fekak H, Rabii R, Moufid K, et al. Cause rare d'anuries obstructives: la tuberculose urogénitale. Annales d'urologie . 2003;37(2):71-74.
14. Dassouli B, Benlemlih A, Joual A, et al. La néphrostomie percutanée en urgence. A propos de 42 cas. Ann Urol. 2001;35(6):305-308.

15. Fournier G, Delavierre D, Le Coat R, et al.La néphrostomie percutanée de drainage chez le patient âgé de plus de 70 ans: A propos de 98 néphrostomies chez 74 patients. Progrès en Urologie. 1994;4:362-370.

16. Allen DJ, Longhorn SE, Philp T, et al.Percutaneous Urinary Drainage and Ureteric Stenting in Malignant Disease. Clin Oncol ( $R$ Coll Radiol) . 2010;22(9):733-739.

17. Sharer W, Grayhack JT, Graham J. Palliative urinary diversion of malignant ureteral obstruction. J Urol . 1978;120(2):162-164.

18. Wilson JR, Urwin GH, Stower MJ. The role of percutaneous nephrostomy in malignant ureteric obstruction. Ann R Coll Surg Engl. 2005;87(1):21-24. 